http://dx.doi.org/10.32911/as.2017.v10.n2.168

Aporte Santiaguino. 10 (2), 2017: 259-270

ISSN 2070-836X

\title{
Sostenibilidad del programa de desarrollo de capacidades en los proyectos productivos de los núcleos ejecutores del microcorredor socioeconómico "Huascarán"-Áncash, 2005-2008
}

\begin{abstract}
Program sustainability of capacity development in the productive projects of executing nuclei of the "Huascaran"-Ancash socioeconomic microcorredor, 2005-2008
\end{abstract}

Mario Villafuerte Vicencio ${ }^{1}$

\section{RESUMEN}

En el período 2005-2008, el Fondo de Cooperación y Desarrollo Social, financió el Programa de Desarrollo de Capacidades (PDCC) en proyectos productivos, bajo la modalidad de núcleos ejecutores (NNEE), en el microcorredor socioeconómico "Huascarán”, Callejón de Huaylas, Áncash-Perú. El PDCC comprendió los ejes temáticos: tecnología productiva, gestión empresarial y articulación al mercado. Los NNEE fueron liquidados, pero sus integrantes optaron por constituirse en asociaciones de productores agropecuarios (AAPPAA), para continuar aplicando el PDCC. De 40 NNEE, 23 se convirtieron en AAPPAA para seguir empleando el PDCC; y el objetivo del presente estudio ha sido evaluar la sostenibilidad del PDCC. Para lo cual, se ha usado la metodología: evaluación de viabilidad y sostenibilidad de proyectos de desarrollo, que comprende 16 indicadores: factores relacionados con el entorno (4), factores relacionados con los actores (5) y factores relacionados con el diseño de intervención (7); y la escala de valoración de 0 a 5, conclusión, de 23 AAPPAA solo 10 (43,5\%) muestran diferentes niveles de sostenibilidad del PDCC: sostenibles (3), potencialmente sostenibles (2) y medianamente sostenibles (5); los 13 (56,5\%) restantes no.

Palabras clave: desarrollo de capacidades; proyectos productivos; asociación de productores agropecuarios; sostenibilidad.

\section{ABSTRACT}

In the 2005-2008 period, the cooperation and Social Development (FONCODES), fund financed program of development of capabilities (PDCC) in the productive projects of breeding of Guinea pigs, peach nectar, ceramic crafts and fabrics, honey,

1 Universidad Nacional Santiago Antúnez de Mayolo. Huaraz, Perú. 
crop production: peach, flower (Carnation), avocado, artichokes, lima, mango, amaranth and corn corn, including the phase of marketing in all projects in the form of executing nuclei (NNEE), in the context of the socio-economic "Huascaran" microcorredor, located in the Callejón de Huaylas, in Áncash-Peru. The PDCC understood the thematic axes: production technology, business management and joint to the market. At the end of the intervention period, the NNEE were liquidated, but members chose to become associations of agricultural producers (AAPPAA), in order to pursue the PDCC of FONCODES, an organized way. The results of the study found that $40 \mathrm{NNEE}$ of productive projects, 23 became AAPPAA and continue using the PDCC in their productive activities. This research work has the purpose of studying the sustainability of the PDCC into the AAPPAA. Which, used the methodology proposed by, which includes the evaluation of 16 indicators grouped into factors related to the environment (4) factors (5) actors and factors related to the design of intervention (7); with rating from 0 to 5 scale. In conclusion, 23 AAPPAA only $10(43.5 \%)$ show different levels of sustainability in the implementation of the PDCC; i.e. sustainable (3), (2) potentially sustainable and moderately sustainable (5); $13(56.5 \%)$ remaining are not.

Keywords: development of skills; productive projects; association of agricultural producers; sustainability.

\section{INTRODUCCIÓN}

A fin de superar la pobreza monetaria rural mediante la generación de ingresos económicos familiares, el Estado implementa programas de proyectos productivos, y no logra sus propósitos debido a la escasa sostenibilidad de dichos programas; convirtiéndose en una permanente preocupación gubernamental. Un antecedente de dichos esfuerzos, es el Programa de Desarrollo de Capacidades (PDCC), ejecutado por el Fondo de Cooperación para el Desarrollo Social (FONCODES) en convenio con el Banco Interamericano de Desarrollo (BID), en proyectos productivos agropecuarios en 11 microcorredores socioeconómicos del país, bajo la modalidad de núcleos ejecutores (NNEE), de 2005 a 2008.

En Áncash, se ejecutaron 40 proyectos productivos en: crianza de cuyes, elaboración de néctar de melocotón, producción de artesanías de cerámica y tejidos, de miel de abeja, cultivo de: melocotón, flores (claveles), palta, alcachofa, lima, mango, kiwicha y maíz choclo, incluyendo las respectivas fases de comercialización, en el Microcorredor Socioeconómico “Huascarán” (MCSE “Huascarán”) -ubicado en el Callejón de Huaylas, entre los distritos de Taricá (Huaraz) y Yuracmarca (Huaylas) - con participación de 1000 productores agropecuarios, agrupados en 40 NNEE. Concluido el período de intervención del PDCC, los NNEE fueron liquidados, y los participantes optaron por transformarse en asociaciones de productores agropecuarios (AAPPAA) para continuar aplicando el PDCC. 
El núcleo ejecutor tiene presidente, secretario, tesorero elegidos por los beneficiaros, y un fiscal que representa al gobierno local, y el PDCC proporciona asistencia técnica, capacitación e información especializada en tecnología productiva, gestión empresarial y articulación al mercado, a los negocios rurales en marcha organizados en NNEE, para solucionar los "cuellos de botella" que tenían los negocios rurales y que individualmente no podían resolverlos.

En consecuencia, el objetivo de esta investigación es determinar la sostenibilidad del PDCC en las AAPPAA, usando como metodología los indicadores de sostenibilidad propuestos por Martínez de Anguita (2006), con sus respectivas escalas de valoración establecidas por Arias-Giraldo y Camargo (2007); para concluir con la identificación de los proyectos productivos y AAPPAA sostenibles en base al empleo del PDCC.

No se ha encontrado trabajos realizados de esta índole, de manera que el presente estudio absolverá la duda existente sobre la sostenibilidad del PDCC, y de hallarse sostenible, servirá como instrumento de orientación para la promoción de negocios rurales con perspectivas de generación de ingresos económicos aplicando el programa mencionado, y constituirá un importante aporte para la reducción de la pobreza monetaria que sufre la población rural; la cual justifica plenamente la realización del estudio.

\section{MATERIALES Y MÉTODOS}

Se empleó el diseño de investigación no experimental, porque solo se analizó el nivel de sostenibilidad del PDCC en las AAPPAA; transversal, porque la recopilación de datos se realizó en un lapso de dos meses (octubre-noviembre 2014); descriptivo, porque se reporta los datos conforme ocurre en el tiempo y espacio; y explicativa, porque se ha tratado de establecer la sostenibilidad del PDCC aplicado por las AAPPAA, (Hernández et al., 2010)

El PDCC de FONCODES, se ejecutó entre 2005 y 2008 en 40 NNEE del MCSE "Huascarán”, cuya sostenibilidad debía evaluarse 7 años después (Martínez de Anguita, 2006), en este caso contados desde 2007, porque 2008 fue el año de liquidaciones de los NNEE. En consecuencia, las unidades de análisis fueron las AAPPAA que seguían aplicando el PDCC en 2014, que en total son 23 (población); y considerando que están ubicados en lugares de fácil accesibilidad y a distancias cortas entre sí, se determinó levantar información de las 23 asociaciones; de manera que la obtención de datos tuvo carácter censal.

Las informaciones primarias, se obtuvieron mediante la aplicación de cuestionarios de encuesta validados con criterios de expertos con una confiabilidad 0,86 de alfa de Cronbach, y usando hojas de entrevista adecuadamente estructuradas. Previa a la realización de la encuesta, se solicitó el consentimiento informado del presidente o miembro(s) de la junta directiva de la asociación, como muestra de respeto a la libertad de proporcionar información, y a fin de propiciar un ambiente apropiado para obtener respuestas veraces que permitan llegar a conclusiones acordes a la realidad, teniendo en cuenta los principios éticos que exige un trabajo de investigación científica. 
El procesamiento de datos se realizó mediante el software estadístico SPSS (v22) previo diseño de tablas estadísticas que respondan a los objetivos y a las hipótesis planteadas.

\section{Análisis de sostenibilidad}

El análisis de sostenibilidad, se efectuó usando los indicadores de Martínez de Anguita (2006) y las escalas de valoración propuesta por Arias-Giraldo y Camargo (2007), que se presentan en las siguientes tablas 1 y 2 , respectivamente.

Tabla 1. Definición resumida de los indicadores de sostenibilidad

\begin{tabular}{|c|c|c|}
\hline \multicolumn{2}{|r|}{ Indicadores } & \multirow{2}{*}{$\begin{array}{l}\text { Definición resumida } \\
\text { Apoyo estatal para generar un entorno político favorable } \\
\text { para la sostenibilidad del proyecto. }\end{array}$} \\
\hline 1 & $\begin{array}{l}\text { Apoyo de las instituciones públicas } \\
\text { al proyecto }\end{array}$ & \\
\hline 2 & $\begin{array}{l}\text { Cuidado del medio ambiente por el } \\
\text { proyecto }\end{array}$ & $\begin{array}{l}\text { Medidas que toma el proyecto para preservar los recur- } \\
\text { sos naturales. }\end{array}$ \\
\hline 3 & $\begin{array}{l}\text { Cambios socioeconómicos genera- } \\
\text { do por el proyecto }\end{array}$ & $\begin{array}{l}\text { Los cambios del proyecto pueden provocar nuevos en- } \\
\text { tornos, afectando incluso al proyecto mismo. }\end{array}$ \\
\hline 4 & $\begin{array}{l}\text { Riesgos del proyecto ante catástro- } \\
\text { fes naturales }\end{array}$ & $\begin{array}{l}\text { El proyecto identifica las áreas de riesgo para reducir la } \\
\text { vulnerabilidad y sus efectos. }\end{array}$ \\
\hline 5 & $\begin{array}{l}\text { Capacidad institucional del pro- } \\
\text { yecto }\end{array}$ & $\begin{array}{l}\text { Los asociados conocen la FODA del proyecto y se sien- } \\
\text { ten involucrados con los objetivos. }\end{array}$ \\
\hline 6 & $\begin{array}{l}\text { Articulación de actores del pro- } \\
\text { yecto }\end{array}$ & $\begin{array}{l}\text { Existen coordinaciones entre los asociados y la Junta } \\
\text { Directiva para lograr los objetivos. }\end{array}$ \\
\hline 7 & $\begin{array}{l}\text { Respeto a los aspectos sociocultu- } \\
\text { rales }\end{array}$ & $\begin{array}{l}\text { Los asociados ejecutan el proyecto respetando los usos y } \\
\text { costumbres de la comunidad. }\end{array}$ \\
\hline 8 & $\begin{array}{l}\text { Equidad de género en la ejecución } \\
\text { del proyecto }\end{array}$ & $\begin{array}{l}\text { Inclusión de las mujeres en todas las actividades produc- } \\
\text { tivas. }\end{array}$ \\
\hline 9 & $\begin{array}{l}\text { Involucramiento de los beneficia- } \\
\text { rios en el proyecto }\end{array}$ & $\begin{array}{l}\text { Los asociados se apropian el proyecto y se erigen como } \\
\text { protagonistas de su desarrollo. }\end{array}$ \\
\hline 10 & $\begin{array}{l}\text { Uso de tecnología apropiada por el } \\
\text { proyecto }\end{array}$ & $\begin{array}{l}\text { Los procesos de transferencia tecnológica impactan en } \\
\text { los asociados y en la localidad. }\end{array}$ \\
\hline 11 & $\begin{array}{l}\text { Acceso a información generada en } \\
\text { la gestión del proyecto }\end{array}$ & $\begin{array}{l}\text { Las informaciones y conocimientos generados son } \\
\text { transferidos a los asociados y a la comunidad? }\end{array}$ \\
\hline 12 & $\begin{array}{l}\text { Comportamiento de los aspectos } \\
\text { económico financieros }\end{array}$ & $\begin{array}{l}\text { Los beneficios se incrementan y compensan económica } \\
\text { y financieramente los costos. }\end{array}$ \\
\hline 13 & $\begin{array}{l}\text { Postura de los asociados ante la } \\
\text { cooperación técnica }\end{array}$ & $\begin{array}{l}\text { Las capacidades individuales y colectivas adquiridas me- } \\
\text { joran la autonomía de los asociados. }\end{array}$ \\
\hline 14 & $\begin{array}{l}\text { Relación entre las acciones y los } \\
\text { recursos del proyecto }\end{array}$ & $\begin{array}{l}\text { Correspondencia entre el alcance del proyecto y los re- } \\
\text { cursos productivos disponibles. }\end{array}$ \\
\hline 15 & $\begin{array}{l}\text { Perspectivas de continuidad del } \\
\text { proyecto }\end{array}$ & $\begin{array}{l}\text { Seguridad de los asociados en la continuidad del proyec- } \\
\text { to, concluido el apoyo externo. }\end{array}$ \\
\hline 16 & Replicabilidad del proyecto. & $\begin{array}{l}\text { Capacidad del proyecto para ser adoptado como "expe- } \\
\text { riencia-tipo", por tanto replicable. }\end{array}$ \\
\hline
\end{tabular}


Tabla 2. Valoración cualitativa de los indicadores e índice de sostenibilidad

\begin{tabular}{lc}
\hline Nivel de Sostenibilidad & Índice de Sostenibilidad \\
\hline Sostenible & $4<\mathrm{x} \leq 5$ \\
Potencialmente sostenible & $3<\mathrm{x} \leq 4$ \\
Medianamente sostenible & $2<\mathrm{x} \leq 3$ \\
Potencialmente insostenible & $1<\mathrm{x} \leq 2$ \\
Insostenible & $0 \leq \mathrm{x} \leq 1$ \\
\hline
\end{tabular}

Asimismo, se construyó diagramas tipo "ameba" o "tela de araña" con los valores obtenidos para visualizar el nivel general de sostenibilidad; precisando que, según Arias-Giraldo y Camargo (2007), mientras más se aproximen los valores de los indicadores al círculo superior (valor 5) el sistema es más sostenible.

Para probar la diferencia significativa entre el valor observado $\left(\mathrm{O}_{j}\right)$ y el valor esperado $\left(E_{j}\right)$, de los indicadores, se empleó la prueba Ji Cuadrada para caso no paramétrico (Siegel, 1990), definida de la siguiente forma:

$$
\chi_{0}^{2}=\frac{\sum_{j=1}^{k}\left(O_{j}-E_{j}\right)^{2}}{E_{j}} \quad \chi_{(k-1)}^{2}
$$

\section{RESULTADOS}

Resultados de las encuestas aplicadas para medir la sostenibilidad

Tabla 3. Unidades de análisis según valoración de sostenibilidad

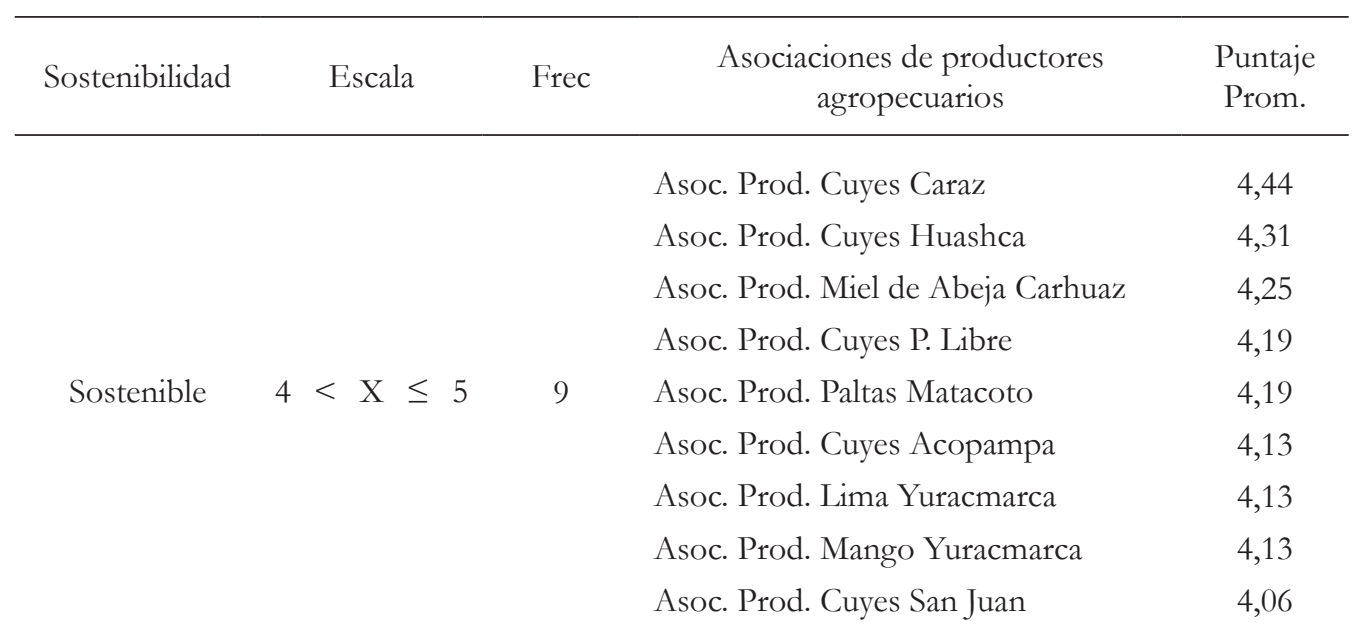




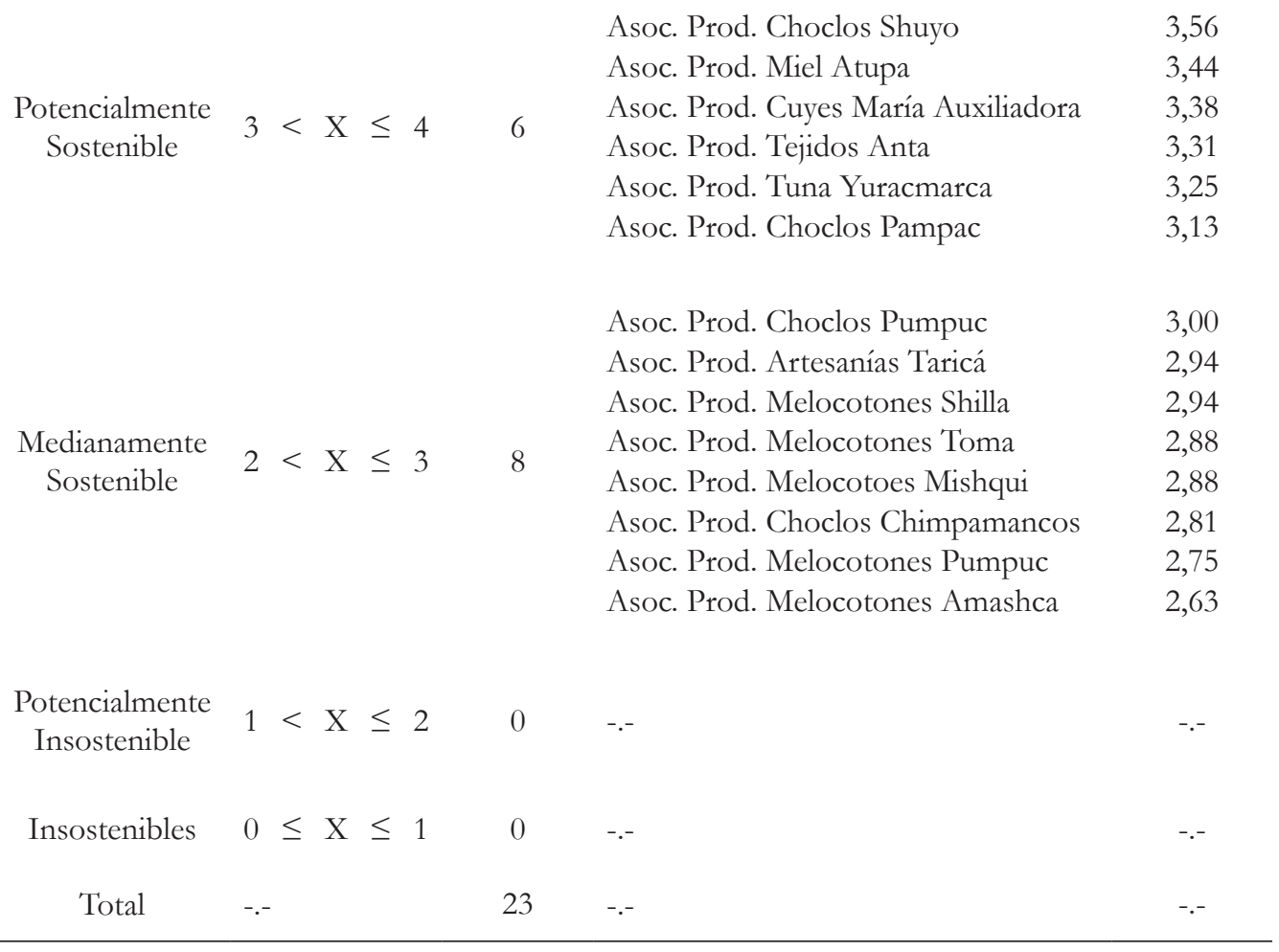

La tabla 3, muestra que 9 (39 \%) asociaciones son sostenibles aplicando el PDCC y estas son: crianza de cuyes (5), productores de miel de abeja (1), palta (1), lima (1) y mango (1); 6 (26\%) potencialmente sostenibles: productores de maíz choclo (2), miel de abeja (1), tunas (1) y tejidos (1): 8 (35\%) medianamente sostenibles: productores de melocotones (5), maíz choclo (2) y artesanías de cerámica (1), y ninguna potencialmente insostenible o insostenible.

\section{Análisis según diagramas tipo "ameba" o "tela de araña"}

A modo de ejemplo, se presenta el diagrama "ameba" de la sostenibilidad del proyecto crianza de cuyes de Caraz. 


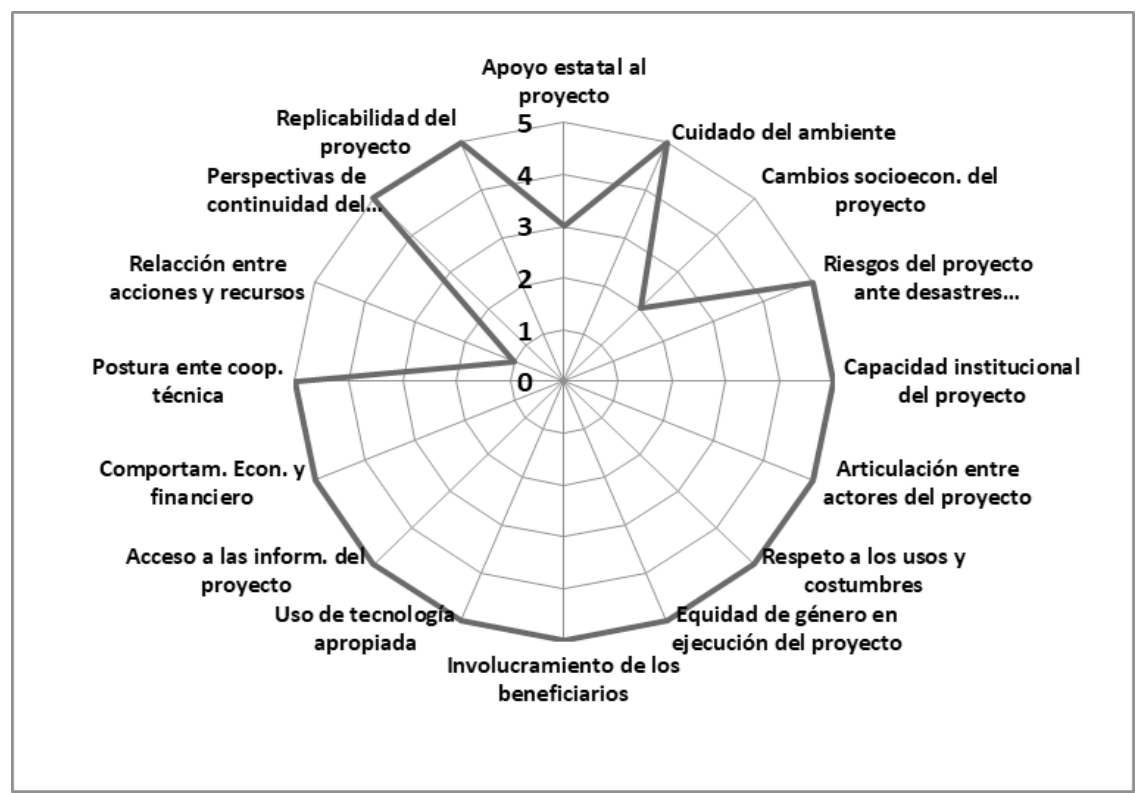

Figura 1. Diagrama de Sostenibilidad del Proyecto de Crianza de Cuyes de Caraz

En la figura 1 se observa que, 13 de 16 indicadores alcanzan la valoración máxima (5) ubicados en el círculo superior, 3 en apoyo estatal, 2 en cambios del entorno socioeconómico, y 1 en la relación entre acciones y recursos; es decir, en este proyecto el PDCC es altamente sostenible porque cuanto más alto sean las valoraciones del proyecto, es más sostenible en ese indicador (Arias-Giraldo y Camargo, 2007). Realizando estos análisis en las 23 asociaciones de productores, se llegó a reafirmar los niveles de sostenibilidad hallados en la tabla 3.

\section{Pruebas de Hipótesis}

Referencialmente se presentan los resultados arrojados por SPSS v22, sobre la sostenibilidad del PDCC en dos proyectos de crianza de cuyes.

Tabla 4. Prueba Ji-Cuadrada para una muestra

\begin{tabular}{|c|c|c|c|c|}
\hline & Hipótesis nula $\left(\mathrm{H}_{\mathrm{o}}\right)$ & Nivel de Signif. $(\alpha)$ & $\mathrm{p}^{*}$ & Decisión \\
\hline 1 & $\begin{array}{l}\text { Las categorías de sostenibilidad del } \\
\text { PDCC en la crianza de Cuyes de Aco- } \\
\text { pampa, ocurren con probabilidades } \\
\text { iguales }\end{array}$ & 0,05 & 0,039 & Signif. \\
\hline 2 & $\begin{array}{l}\text { Las categorías de sostenibilidad del } \\
\text { PDCC en la crianza de Cuyes "San } \\
\text { Juan", ocurren con probabilidades } \\
\text { iguales }\end{array}$ & 0,05 & 0,646 & No Signif. \\
\hline
\end{tabular}

*Se muestran significaciones asintóticas 
Como $\mathrm{p}<\alpha$, se rechaza la hipótesis nula; lo cual indica que las valores altos son significativamente superiores a los valores bajos, revelando que el proyecto crianza de cuyes de Acopampa es sostenible, mientras que la crianza de cuyes "San Juan", no es sostenible.

Realizando la prueba para las 23 asociaciones de productores, se encontró sostenibilidad significativa de 10 (43,5 \%) proyectos: sostenibles: crianza de cuyes de Caraz y de Acopampa, apicultores de Carhuaz; potencialmente sostenibles: Crianza de cuyes "María Auxiliadora" y cultivo de tunas de Yuracmarca; medianamente sostenibles: Artesanías de cerámica de Taricá, cultivo de melocotones de Shilla, Toma y Pumpuc y maíz choclo de Chimpamancos; y sostenibilidad no significativa en los restantes $13(65,5 \%)$ proyectos.

La siguiente tabla, muestra los resultados consolidados:

Tabla 5. Consolidado de AAPPAA por nivel de significancia según sostenibilidad

\begin{tabular}{|c|c|c|c|}
\hline $\begin{array}{l}\text { Sosteni- } \\
\text { bilidad }\end{array}$ & $\begin{array}{l}\text { Nivel de significancia } \\
\text { Significativa }\end{array}$ & No significativa & Total \\
\hline 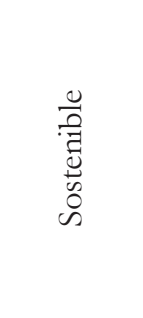 & $\begin{array}{l}\text { - Crianza de Cuyes-Caraz } \\
\text { - Asoc. de Apicultores-Carhuaz } \\
\text { - Crianza de cuyes-Acopampa }\end{array}$ & $\begin{array}{l}\text { - Crianza de cuyes-Huashca } \\
\text { - Crianza de cuyes-P. Libre } \\
\text { - Cultivo de paltas-Matacoto } \\
\text { - Cultivo de Lima-Yuracmarca } \\
\text { - Cultivo de Mango-Yuracmarca } \\
\text { - Crianza de cuyes San Juan-Shilla }\end{array}$ & 9 \\
\hline 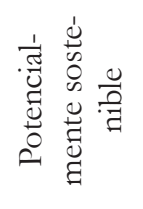 & $\begin{array}{l}\text { - Crianza de cuyes María Auxiliadora-Shilla } \\
\text { - Cultivo de Tunas-Yuracmarca }\end{array}$ & $\begin{array}{l}\text { - Cultivo de Choclo-Shuyo } \\
\text { - Miel de abeja-Atupa } \\
\text { - Asoc. de tejidos-Anta } \\
\text { - Cultivo de Choclo-Pampac }\end{array}$ & 6 \\
\hline 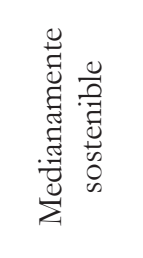 & $\begin{array}{l}\text { - Artesanías de cerámica-Taricá } \\
\text { - Cultivo de melocotones-Shilla } \\
\text { - Cultivo de Melocotones-Toma } \\
\text { - Cultivo de Choclo-Chimpamancos } \\
\text { - Cultivo de melocotones-Pumpuc }\end{array}$ & $\begin{array}{l}\text { - Cultivo de choclo-Pumpuc } \\
\text { - Cultivo de melocotones-Mishqui } \\
\text { - Cultivo de meloctones-Amashca }\end{array}$ & 8 \\
\hline Total & 10 & 13 & 23 \\
\hline
\end{tabular}




\section{DISCUSIÓN}

La discusión está centrada en el análisis de la sostenibilidad del PDCC en cada uno de los proyectos identificados como significativamente sostenibles dentro de los respectivos niveles de sostenibilidad (tabla 5), agrupándolos si pertenecen a la misma línea de actividad.

1. Proyectos de Crianza y Comercialización de Cuyes de Caraz, Acopampa y María Auxiliadora (Distrito Shilla). De estos tres proyectos, el de Caraz y Acopampa son sostenibles, mientras que "María Auxiliadora" es potencialmente sostenible, de acuerdo a los puntajes alcanzados, (Arias-Giraldo y Camargo, 2007). La crianza y venta de cuyes es una actividad ancestralmente practicada por mujeres del área rural como parte de su actividad doméstica, en "cuyeros" acondicionados junto a las cocinas de sus viviendas, de manera que la sostenibilidad de estos proyectos, en parte pueden atribuirse a estas costumbres. Según las personas encuestadas, el PDCC ha incrementado la participación de las mujeres, convirtiéndolas en organizaciones fuertes (Tello et al., 2000), con plena identificación con sus proyectos y motivadas al ver que su contribución a los ingresos familiares es trascendente, que se refleja en frases como, "antes dependíamos mucho de nuestros maridos, ahora aportamos iguales"; tienen mayor predisposición para coordinar y realizar acciones conjuntas (Abric, 2001), ponen en práctica las tecnologías que aprenden en las capacitaciones y las transfieren a las mujeres no asociadas, se sienten satisfechas con las utilidades económicas de sus proyectos, aseguran que continuarán con esta labor con o sin apoyo externo, y replican sus aprendizajes en granjas propias (Triveño et al., 2011); complementariamente, los desechos de los cuyes son utilizados para el abonamiento de las tierras de cultivo y plantas, conservando así este importante recurso, y la cercanía de los factores productivos (granja-pastura) reducen los costos de producción (Vargas, 2002).

2. Asociación de Productores de Miel de Abeja de Carhuaz (API Carhuaz). Acumularon valoración 5, en 5 indicadores, 4 en 10 y 3 en 1; es decir, el PDCC es sostenible (Arias-Giraldo y Camargo, 2007). Los apicultores afirman que se capacitan constantemente en tecnologías de producción y articulación al mercado para diversificar productos y elevar sus ingresos, coordinan bien sus actividades (articulación entre actores), laboran con autonomía individual y colectiva, y replican con empeño el PDCC (Triveño et al., 2011). Es potencialmente sostenible en la creación de un ambiente político favorable para conseguir el apoyo estatal, esta actividad no utiliza ninguna sustancia que contamine el ambiente, las colmenas están ubicadas en lugares más o menos seguros, la picadura de la abeja es empleada para curar el reumatismo, consideran que la apicultura les asegura el futuro, y transfieren sus experiencias a la comunidad; y es medianamente sostenible porque no siempre transfieren las informaciones acumuladas a otros apicultores (Machado, 2000). 
3. Proyecto de cultivo y comercialización de Tunas de Yuracmarca. Es un proyecto potencialmente sostenible. Los asociados sostienen que sus fortalezas están en la participación activa de las mujeres (Tello et al., 2000), y en la visibilización del cultivo de tunas aprovechando terrenos eriazos y mejorando el entorno físico del distrito de Yuracmarca, contribuyen a la conservación ambiental por ser un cultivo nativo, consideran relativamente vulnerable porque se cultivan en áreas propensas al desprendimiento de rocas, admiten que la coordinación entre asociados y la junta directiva debe fortalecerse, se sienten motivados al haber abierto una nueva línea de negocios, las tecnologías adoptadas para el cultivo y cosecha aún están en prueba, que el PDCC eleva sus autonomías, la escasez del agua pone en cierto riesgo el proyecto, aseguran que darán continuidad al proyecto aun cuando el Estado no muestra interés en la promoción de esta actividad (Iglesias, 2006), y que es una actividad fácilmente replicable.

4. Proyecto Elaboración y Comercialización de Artesanías de Cerámica de Taricá. Está clasificado como un proyecto medianamente sostenible. Cuenta con infraestructuras y equipos dotados por el Estado. Los artesanos manifiestan que, para lograr sus objetivos procuran producir y vender conjuntamente, socializan sus informaciones, al extraer la arcilla tienen cuidado ambiental, el centro artesanal por su ubicación atenúa los riesgos; tratan de preservar la identidad local y regional mediante el arte popular, tienen habilidades para producir artesanías utilitarias, adaptan tecnologías para mejorar los acabados y transfieren al exterior, que es la fuente principal de ingresos económicos (tiende a decrecer), la continuidad del proyecto es segura y replicable por ser una actividad ancestral. Tienen debilidades en la comercialización de sus productos artesanales por la presencia masiva de productos provenientes de Puno y otras regiones, consideran necesaria reorientar su organización y fortalecerse internamente para elevar la institucionalidad (Machado, 2000).

5. Proyectos de Cultivo y Comercialización de Melocotones de Shilla, Toma y Pumpuc (Tinco). Son medianamente sostenibles y con características similares entre sí y están localizados en la microcuenca Buin (provincia de Carhuaz). El cultivo de melocotones tiene alta incidencia provincial, el Estado apoya con infraestructuras de acceso y riego. Los productores indican que, los plantones tienden a infectarse motivando el uso excesivo de insecticidas y de abonos no orgánicos, sin embargo, resulta más rentable que otros cultivos; adquieren destrezas necesarias como cultivar individual e independientemente, la existencia suficiente de suelo y agua reducen los costos y es replicable aplicando el PDCC. Intervienen por igual varones como mujeres, se sienten artífices de la mejora social conjunta, adaptan nuevas tecnologías mediante capacitaciones, comparten sus experiencias e institucionalmente se consolidan al formar parte de organizaciones productivas de nivel provincial (Machado, 2000). 
6. Proyecto Cultivo y Comercialización de Choclo de Chimpamancos (Mancos). Es un proyecto medianamente sostenible. Según estos agricultores, sobresalen en equidad de género, reafirman que el cultivo de choclo es una actividad de mayor incidencia en la zona, su incremento o disminución en áreas sembradas es monitoreada por el sector agricultura, sin embargo, consideran que el apoyo público es muy limitado, les mortifica vender sus choclos a precios reducidos impuestos por los compradores intermediarios, admiten que coordinan en la fase de cultivo más no en la comercialización, en cierta medida realizan labores agrícolas de corte tradicional, se apropian moderadamente de sus proyectos, se "desenganchan" sin inconvenientes del apoyo externo, la transferencia tecnológica es limitada, aun cuando las utilidades son decrecientes continuarán cultivando con miras a superar la pobreza (Kliksberg, 1999), admiten que la minifundización reduce el rendimiento económico, y la debilidad está en el sobreuso de insecticidas.

\section{CONCLUSIONES}

De los 40 proyectos productivos ejecutados en el microcorredor socioeconómico "Huascarán” bajo la modalidad de núcleos ejecutores, solo 23 conformaron asociaciones de productores agropecuarios y continúan aplicando el programa de desarrollo de capacidades; 10 de ellos son sostenibles, potencialmente sostenibles o medianamente sostenibles; y los otros 13 no lo son.

Los proyectos sostenibles son: crianzas de cuyes de Caraz y Acopampa y producción de Miel de Abeja de Carhuaz; potencialmente sostenibles: crianza de cuyes "María Auxiliadora" (distrito Shilla) y cultivo de Tunas de Yuracmarca; medianamente sostenible: artesanías de cerámica de Taricá, producción de melocotones de Shilla, Toma y Pumpuc (distrito Tinco), y cultivo de choclo de chimpamancos (distrito Mancos).

Los indicadores que sustentan dicha sostenibilidad del PDCC específicamente son: la equidad de género, las fortalezas en participación y articulación de los actores, los aspectos económicos y financieros satisfactorios, la protección medioambiental y la replicabilidad de los proyectos.

\section{REFERENCIAS BIBLIOGRÁFICAS}

Abric, Jean-Claude. 2001. «Prácticas Sociales y Representaciones». Revista Filosofía y Cultura Contemporánea $\mathrm{N}^{\circ}$ 16. México D.F.: Ediciones Coyoacán. Cap. I

Arias-Giraldo, Ligia y Camargo, Juan. 2007. Análisis de sustentabilidad en unidades productivas ganaderas del municipio de Circasia (Quindío - Colombia), Cuenca del Río La Vieja. Colombia: Universidad Tecnológica de Pereira. 
Hernández, Roberto; Fernández, Carlos y Baptista, María del Pilar. 2010. Metodología de la investigación. 5ta. ed. México D.F.: McGraw-Hill/Interamericana Editores.

Iglesias, Enrique. 2006. «El papel del Estado y los paradigmas económicos en América Latina» Revista de la CEPAL 90-Diciembre. Santiago de Chile: CEPAL. 7-15.

Kliksberg, Bernardo. 1999. «Seis tesis no convencionales sobre participación». Revista de estudios sociales. $\mathrm{N}^{\circ}$ 4. Bogotá: Universidad Los Andes.

Machado, Absalón. 2000. El papel de las organizaciones en el desarrollo rural. Bogotá: Pontificia Universidad Javeriana.

Martínez de Anguita, Pablo. 2006. Organización y gestión de proyectos. Publicación académica. Madrid: Universidad Rey Juan Carlos. Tema 6.

Siegel, Sidney. 1990. Estadística no paramétrica. Aplicada a las ciencias de la conducta. 3a. ed. México: Trillas.

Tello, Griselda; Valdivia, Betsey y Valverde, Lourdes. 2000. «Aportes para un programa de formación para el trabajo», Cap. 10. En Portocarrero, Felipe (Comp.). Políticas Sociales en el Perú: Nuevos Aportes. Lima: Red para el Desarrollo de Ciencias Sociales. 349-386.

Triveño, Gladys y otros. 2011. Buenas Prácticas para el desarrollo de la cadena Productiva de la Papa: Experiencias con el Proyecto INCOPA en el Perú. Lima: Centro Internacional de la Papa.

Vargas, Jorge. 2002. Políticas Públicas para la Reducción de la Vulnerabilidad frente a los Desastres Naturales y Socioculturales. New York: CEPAL.

Recibido: 22/07/17

Aceptado: 27/10/17

\section{Correspondencia}

Mario C. Villafuerte Vicencio

mcvillafuertev@gmail.com 